BMJ Open

Diabetes

Research

\& Care

\title{
Projected burden of type 2 diabetes mellitus-related complications in Singapore until 2050: a Bayesian evidence synthesis
}

\author{
Ken Wei Tan (1) , Borame Sue Lee Dickens, Alex R Cook
}

To cite: Tan KW, Dickens BSL, Cook AR. Projected burden of type 2 diabetes mellitusrelated complications in Singapore until 2050: a Bayesian evidence synthesis. BMJ Open Diab Res Care 2020;8:e000928. doi:10.1136/ bmjdrc-2019-000928

- Additional material is published online only. To view please visit the journal online (http://dx.doi.org/10.1136/ bmjdrc-2019-000928).

Received 25 September 2019 Revised 16 January 2020 Accepted 9 February 2020

\section{Check for updates}

\section{(c) Author(s) (or their} employer(s)) 2020. Re-use permitted under CC BY-NC. No commercial re-use. See rights and permissions. Published by BMJ.

Saw Swee Hock School of Public Health, National University of Singapore and National University Health System, Singapore

Correspondence to Dr Alex R Cook; ephcar@nus.edu.sg

\section{ABSTRACT}

Objective We examined the effects of age, gender, and ethnicity on the risk of acute myocardial infarction, stroke, and end-stage renal disease according to type 2 diabetes mellitus status among adults aged 40-79 in Singapore. Methods A Bayesian inference framework was used to derive age-specific, gender-specific and ethnicity-specific prevalence of type 2 diabetes mellitus from the 2010 Singapore National Health Survey, and age-standardized gender and ethnicity-specific incidence rates of acute myocardial infarction, stroke and end-stage renal disease from the National Registry of Diseases Office. Population forecasts were used in tandem with incidence rates to project the future chronic disease burden until 2050. Results The highest relative risk of acute myocardial infarction was observed in the youngest age group (aged 40-44), with higher relative risk for women (men: 4.3 (2.7-6.4); women: 16.9 (9.3-28.3)). A similar trend was observed for stroke (men: 6.5 (4.2-9.7); women: 10.7 (6.0-17.4)). For end-stage renal disease, the highest relative risk was for men aged 45-50 (11.8 (8.0-16.9)) and women aged 55-60 (16.4 (10.7-24.0)). The annual incidence of acute myocardial infarction is projected to rise from 9300 (in 2019) to 16400 (in 2050), the number of strokes from 7300 to 12800 , and the number of end-stage renal disease cases from 1700 to 2700 .

Conclusions Type 2 diabetes mellitus was associated with an increased risk of complications and is modulated by age and gender. Prevention and early detection of type 2 diabetes mellitus can reduce the increasing burden of secondary complications.

\section{INTRODUCTION}

Type 2 diabetes mellitus (DM) is one of the leading, non-communicable causes of premature death and disability. ${ }^{1}$ In 2010, an estimated $7.4 \%$ of the population in England had DM, with the proportion expected to increase to $9.5 \%$ by $2030 .{ }^{2}$ Germany is experiencing the same phenomenon, with the prevalence of DM expected to rise from $10.5 \%$ in 2010 to $16.3 \%$ in $2040 .{ }^{3} \mathrm{DM}$ dramatically increases an individual's risk of developing sequelae. A cohort study on 3020 patients with lacunar stroke reported $37 \%$ with DM at baseline. ${ }^{4}$ A study on 832 individuals who

\section{Significance of this study}

What is already known about this subject?

- With an aging population, Singapore is projected to have up to one million individuals with diabetes by 2050.

- As individuals with diabetes are more prone to developing complications, the number of acute myocardial infarction, stroke and end-stage renal disease is also expected to rise.

What are the new findings?

- There are highly differential incidence rates of acute myocardial infarction, stroke and end-stage renal disease between genders, ethnicities, and those with and without diabetes.

- The incidence of acute myocardial infarction, stroke and end-stage renal disease was projected up to 2050.

How might these results change the focus of research or clinical practice?

- Stratified incidence of these chronic diseases helps to understand the differential rates of developing complications by demographics and diabetic status.

- Projections of chronic disease burden will help policy planners to better estimate the growing healthcare needs of the nation and ensure adequate amount of care is provided.

were hospitalized for acute myocardial infarction (AMI) reported a DM prevalence of $9.7 \% .^{5} \mathrm{DM}$ is also a major risk factor of endstage renal disease (ESRD). In 2013, 24\% of incident renal replacement therapy users as a result of ESRD had DM as the primary cause of ESRD. ${ }^{6}$ These sequelae of DM will impose an increasing burden on individuals and health systems. Global estimates of the economic burden of DM in 2015 were approximately US $\$ 1.31$ trillion, $1.8 \%$ of the global gross domestic product. ${ }^{7} \mathrm{DM}$ prevalence is high in those aged 45 and above, although there is a rising trend of individuals aged 30-39 being diagnosed. ${ }^{8}$ Those with early-onset DM below 
age 45 have a 14-fold greater risk of developing AMI as compared with the fourfold risk for those above age $45 .{ }^{9}$ Given the expected increase in DM prevalence, this figure will correspondingly rise, with a significant proportion attributable to sequelae.

The greater susceptibility to DM of the Asian genotypes-the recent prevalence being $10.9 \%$ in China, ${ }^{10}$ $17.5 \%$ in Malaysia, ${ }^{11} 9.9 \%$ in Thailand, ${ }^{12}$ and $5.7 \%$ in Indonesia, ${ }^{13}$ although rates are high throughout the region-further exacerbates these trends in the most populous continent. Within South-East Asia, multiethnic Singapore is primarily composed of Chinese, Malays and Indian ethnicities and is therefore expected to face high complication rates with the rapidly increasing prevalence of DM from $2 \%$ in 1975 to $11 \%$ in $2010^{14}$; this prevalence is projected to reach one in six adults by $2050 .{ }^{15}$ Interethnic differences in the risk of diabetic complications are known to exist ${ }^{16}$ and warrant some exploration in the context of Singapore. In the UK, significant disparities were identified between major ethnic groups in terms of DM prevalence and associated chronic kidney disease, with South Asians exhibiting higher prevalence of DM and more severe kidney disease relative to the Caucasian population. ${ }^{17}$

As in many countries, Singapore's population is aging, and the proportion of individuals aged 60 and above is expected to rise from $13.3 \%$ in 2010 to $31.9 \%$ in 2050 , making it a superaged country. At a population level, the rapidly aging population and low mortality rates will increase the proportion of people living with DM, which will drastically increase the number of individuals at risk of developing sequelae. The prevalence of DM in Singapore for citizens and permanent residents aged 18-69 rose from $7.3 \%$ in 1992 to $8.6 \%$ in $2017,{ }^{18}$ and the International Diabetes Federation estimates a DM prevalence of $13.7 \%$ for the entire adult population, inclusive of foreigners. ${ }^{19}$ In response to the rising prevalence of DM, the Singapore government in 2016 declared 'war' on diabetes, ${ }^{20}$ leading to a multipronged, nationwide response. To guide effective policy planning and interventions, estimates of the burden of DM are required. We therefore aim to assess the combined effects of age, gender and ethnicity on the risk for three main morbidities associated with DM-AMI, stroke, and ESRD—stratified by DM status among adults aged 40-79 in Singapore, using Bayesian evidence synthesis to pool information from cross-sectional and registry databases, and to project forward to 2050 using an individual-level model.

\section{METHODS}

This analysis had two outputs for AMI, stroke and ESRD: incidence rates and disease incidence projections from 1990 to 2050 . The first step was to estimate DM prevalence by age, gender and ethnicity strata, derived from the 2010 National Health Survey data, ${ }^{14}$ in order to calculate the proportion of each stratum that had DM or was normoglycemic. For ethnicities we considered Chinese,
Malays and Indians, which together comprise $97 \%$ of the population. ${ }^{21}$ We also obtained data from national disease registries containing all reported national cases of AMI, stroke and ESRD. Using Markov chain Monte Carlo simulation, ${ }^{22}$ we estimated the incidence rates for AMI, stroke and ESRD overall and stratified by age, gender, ethnicity and DM status (DM, non-DM). Overlaying the incidence rates with population projections stratified by age, gender and ethnicity from 1990 to $2050,{ }^{15}$ we validate our model against empirical registry data ranging from 2007 to 2016 (AMI), from 2005 to 2016 (stroke), and from 1999 to 2015 (ESRD), and project forward to 2050 to estimate the future disease burden of these three sequelae.

\section{DM prevalence}

DM prevalence was derived from the 2010 National Health Survey, ${ }^{14}$ a population-based, cross-sectional and multistage survey conducted by the Ministry of Health, Singapore, that provides information on the health profile of the Singapore resident population and their use of healthcare services. DM status was determined based on medical history of known diagnoses and a plasma glucose 2-hour postoral glucose tolerance test value of $\geq 11.1 \mathrm{mmol} / \mathrm{L}$ for newly diagnosed cases, following the diagnostic criteria of the 2010 National Health Survey.

To derive age-specific, gender-specific and ethnicityspecific prevalence of DM, we developed a model of the prevalence $\left(p_{\text {age }}\right)$ inclusive of previously diagnosed and newly diagnosed DM. A Bayesian hierarchical model was developed to estimate the prevalence by 1-year age groups $(a)$, gender $(g)$, and ethnicity $(e)$. With $\theta_{\text {age }}$ as the logit transform of $p_{\text {age }}$, a Gaussian random walk was employed to model $\theta_{\text {age }}$ conditionally:

$$
\theta_{a g e} \mid \theta_{a-1, g e} \sim N\left(\theta_{a-1, g e}, \sigma_{g e}^{2}\right)
$$

where $\sigma_{g e}^{2}$ is an estimated tuning parameter that determines the degree of smoothness of prevalence with age.

\section{AMI, stroke and ESRD incidence}

Data held in the Singapore Myocardial Infarction Registry, Singapore Stroke Registry, and the Singapore Renal Registry are routinely collected and analyzed by the National Registry of Diseases Office to evaluate secular trends and patterns and to support healthcare service planning. ${ }^{23}$ The Singapore Myocardial Infarction Registry contains data of all AMI episodes in all private and public hospitals and a small number of AMI deaths that occurred at home, and was constructed from hospital inpatient discharge summaries, cardiac biomarker trend lists, national medical claims listings and death registry data. The Singapore Stroke Registry data only included stroke episodes diagnosed in public healthcare institutions (where the majority of patients with stroke receive care), and were derived from a combination of national medical claims listings, hospital inpatient discharge summary reviews, and the same death registry data. The 
Table 1 Summary of characteristics of individuals from the Singapore Myocardial Infarction Registry, Singapore Stroke Registry and Singapore Renal Registry

\begin{tabular}{llll}
\hline & $\begin{array}{l}\text { Singapore } \\
\text { Myocardial } \\
\text { Infarction }\end{array}$ & $\begin{array}{l}\text { Singapore } \\
\text { Stroke } \\
\text { Registry }\end{array}$ & $\begin{array}{l}\text { Singapore } \\
\text { Renal } \\
\text { Registry }\end{array}$ \\
\hline Duration & $2007-2016$ & $2005-2016$ & $1999-2015$ \\
\hline Age range & $40-99$ & $40-99$ & $40-99$ \\
\hline $\begin{array}{l}\text { Number of } \\
\text { cases }\end{array}$ & 83969 & 72952 & 19083 \\
\hline Men (\%) & 64.9 & 56.4 & 52.4 \\
\hline $\begin{array}{l}\text { Chinese (\%) } \\
\text { Malay (\%) }\end{array}$ & 66.6 & 76.8 & 70.2 \\
\hline $\begin{array}{l}\text { Indian (\%) } \\
\text { Riabetes (\%) }\end{array}$ & 19.2 & 14.9 & 21.6 \\
\hline $\begin{array}{l}\text { Unknown } \\
\text { diabetic status } \\
\text { (\%) }\end{array}$ & 2.5 & 6.4 & 6.9 \\
\hline
\end{tabular}

Singapore Renal Registry data were collected from all healthcare institutions in Singapore providing care for patients with ESRD via mandatory case notifications. Potential cases of ESRD had their estimated glomerular filtration rate monitored for 6 months to confirm the diagnosis of ESRD using the patient's case notes and electronic medical records. Together, these national registry databases provide epidemiologic and clinical data of annual diagnosed AMI, stroke and ESRD cases among the resident population of Singapore.

Aggregated data on annual AMI (2007-2016), stroke (2005-2016) and ESRD (1999-2015) cases by 5-year age groups for those aged 40-79, gender, ethnicity, and DM status were extracted from the three national disease registries (table 1). The 5-year age groups were used as there were too few individuals to segregate strata by 1-year age groups to obtain stable estimates of incidence. To estimate the annual incidence of AMI, stroke and ESRD—stratified by 5 -year age groups, gender, ethnicity, and DM status-we used a binomial model without secular trends:

$$
y_{\text {age }}^{d}(t) \sim \operatorname{Bin}\left(N_{\text {age }}^{d}(t), \pi_{\text {age }}^{d}\right)
$$

where $y_{\text {age }}^{d}(t)$ denotes counts of disease cases (AMI/ stroke/ESRD) at time $t, N_{a g e}^{d}(t)$ denotes estimated population counts at time $t$ using population data and the model of DM prevalence, and $\pi_{a g e}^{d}$ denotes disease incidence of age group $a$, ethnic group $e$, gender $g$ and DM status $d$. The disease incidence for individuals with DM divided by the disease incidence for non-DM individuals is the relative risk (RR). Combining the estimated DM prevalences with the incidence rates and the 2015 midyear estimates of population structure from the Department of Statistics, Singapore gives the age-standardized incidence rates
(ASIRs) for each population stratum by gender, ethnicity and DM status.

\section{Disease burden estimates}

Population structure of each 1-year age group, gender and ethnicity stratum was obtained from a previous study for the years 1990-2050. ${ }^{15}$ Multiplying the number in each stratum with estimated DM prevalence gives the number of individuals in each stratum with and without DM. Combining these with the incidence rates of each stratum of the population for each year, we obtained the expected total incident cases of each sequelae by year, as well as the number of expected DM incident cases. The incidence of each sequelae for each 5-year age group was applied to each of the 1-year age groups within. Comparison of the expected total and DM incident cases with the empirical incident cases provides internal validation for the model.

\section{Implementation}

The full Bayesian model was implemented using JAGS V.4.3.0. ${ }^{24}$ We used a weakly informative exponential prior, $\operatorname{Exp}(1)$, on the smoothing parameter $\sigma_{g e}$ and uniform non-informative priors for other parameters. Data preparation and analysis were done in $\mathrm{R},{ }^{25}$ and JAGS was called from $\mathrm{R}$ using the package rjags using four independent chains of 1000000 iterations each, with a burn-in of 10000 and thinning of 100 . Convergence was assessed by trace and posterior density plots for all parameters. All estimates provided were posterior medians with $95 \%$ equal-tailed credible intervals (CI). The Bayesian posterior hypothesis probabilities $\tilde{p}$ are the posterior probability that a hypothesis is true, derived from the retained Markov chain Monte Carlo iterations. We performed sensitivity analyses as some individuals had unknown DM status (AMI: $2.5 \%$; stroke: $32.1 \%$; ESRD: $0.3 \%$ ), and reran the analyses assuming all individuals with missing DM status were either DM or non-DM.

\section{RESULTS}

\section{DM prevalence}

The estimated prevalence of DM by age, gender, and ethnicity using the 2010 National Health Survey data is presented in figure 1 . The total prevalence of DM was overall higher for men aged 40 at $11.3 \%$ (95\% CI 6.8\%$18.5 \%)$ than women at $6.5 \%(95 \%$ CI $3.5 \%-11.9 \%)$. DM prevalence increases with age to $48.0 \%(28.2 \%-70.8 \%)$ and $33.6 \%(19.4 \%-54.9 \%)$ for men and women aged 79 , respectively. Age-specific prevalence rates of DM varied considerably by ethnicity, especially among the elderly. The estimated prevalence of DM for those aged 79 was 2.35 times higher among Malay and Indian women $(64.9 \% \quad(41.0 \%-84.0 \%)$ and $64.8 \% \quad(36.8 \%-86.1 \%)$, respectively) than their Chinese counterparts, at $27.5 \%$ $(10.7 \%-52.8 \%)$. This disparity was less pronounced among men, with the prevalence of DM for Malay and Indian men being 1.28 and 1.7 times higher $(57.6 \%$ 
Male DM prevalence

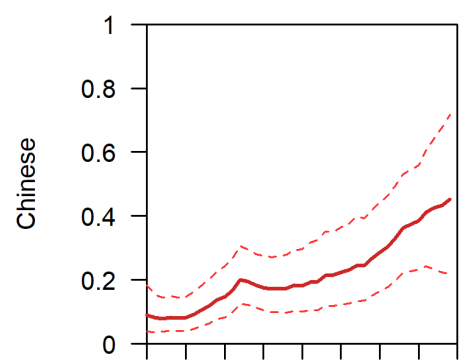

Female DM prevalence
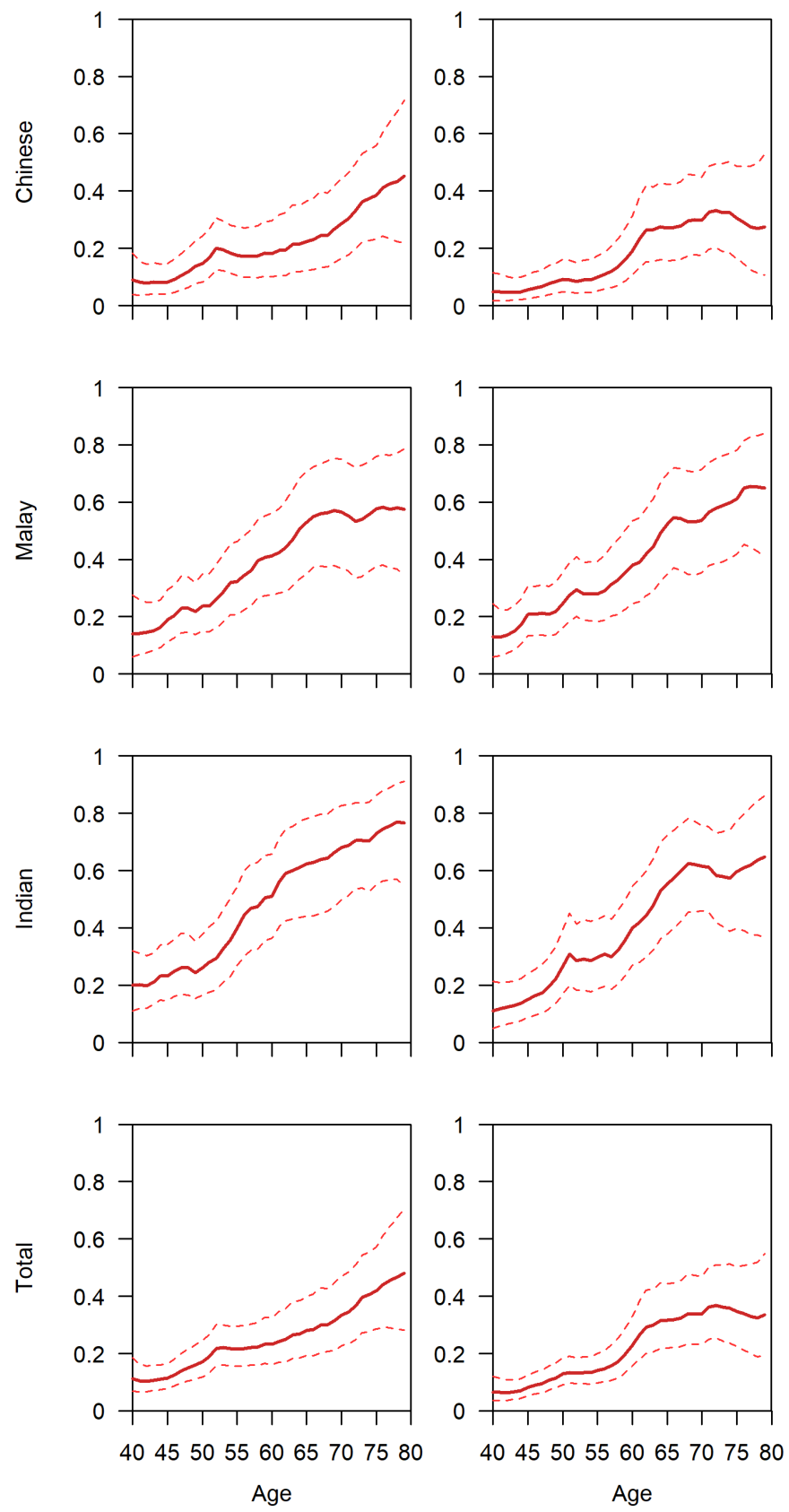

Figure 1 Prevalence estimates of diabetes mellitus (DM) by age, gender and ethnicity for Singaporeans aged 40-79 using data from the 2010 Singapore National Health Survey.

$(34.1 \%-78.5 \%)$ and $76.7 \%(54.4 \%-91.0 \%)$, respectively) than Chinese men, at $45.1 \%(21.9 \%-71.6 \%)$.

\section{RR of AMIl, stroke and ESRD}

For the youngest age group of 40-44, the RR of AMI for men and women with DM was 4.3 (2.7-6.4) and 16.9 (9.328.3) times that of the population without DM (figure 2), illustrating the disparity in RRs between genders. By age 79 , this risk disparity diminished to $1.3(0.6-2.3)$ and 3.4 (1.7-6.0). For almost all combinations of gender and ethnicity (online supplementary figure S4), the risk of AMI among individuals with DM converged with those without DM as age increased, and the RRs were statistically not distinguishable by the highest age group. The estimates did not differ substantially in sensitivity analyses when all individuals with AMI and unknown DM status were reclassified as DM or non-DM (online supplementary figure S1).

Similar but less pronounced trends were observed for stroke. RR of men and women with DM aged 40-44 was 6.5 (4.2-9.7) and 10.7 (6.0-17.4) (figure 2) times higher than their non-DM peers. RR decreased with age to 2.2 (1.1-3.9) and 4.2 (2.2-7.2) for men and women aged 75-79. Less convergence was observed for the various ethnicity and gender combinations in the oldest age group as compared with AMI (online supplementary figure S5). Estimates for stroke were sensitive to how those with unknown DM status were classified, due to more missingness (online supplementary figure S2).

ESRD displayed a slightly different trend in RR, with a peak RR of 11.8 (8.0-16.9) for men with DM at 45-50 years of age, and a value of 16.4 (10.7-24.0) for women at 55-60 years of age (figure 2). Many of the ethnicity and gender combinations did not have the highest RR in the lowest age group of 40-44 like the other sequelae (online supplementary figure S6). This is likely due to chronic kidney disease having different stages of progression, with stage 5 denoting ESRD and the highest rate of progression to stage 5 in the middle-aged. In the elderly, the RR attenuates to 1.9 (0.9-3.4) and 5.3 (2.6-9.4) for men and women aged 75-79. As with AMI, the estimates were robust as it did not matter whether those with unknown DM status were reclassified as having DM or not (online supplementary figure S3).

\section{Age-standardized incidence rates}

ASIRs of AMI, stroke and ESRD were significantly higher among those with DM for all demographic strata (figure 3). Men also tended to have higher rates than women after accounting for DM status. As with the RR estimates, the ASIRs for stroke but not AMI or ESRD were sensitive to how unknown DM status was accounted for (online supplementary figure S7). Marked ethnic differences in ASIRs were observed for all three chronic conditions (figure 3). For non-DM individuals, the ASIR of AMI was roughly twice as high in Malays (men: 7.3 (6.78.0); women: 1.7 (1.5-1.8) per 1000 person-years) and Indians (men: 6.0 (5.5-6.6); women: 1.2 (1.1-1.4) per 1000 person-years) than in Chinese (men: 3.3 (3.2-3.5); women: $0.8(0.8-0.9)$ per 1000 person-years) for both men and women. In individuals with DM, this effect was less pronounced, as ASIRs of AMI were approximately 50\% larger in Malays (men: 17.3 (14.9-20.7); women: $11.2(9.7-13.1)$ per 1000 person-years) and Indians (men: 15.6 (13.7-18.1); women: 11.3 (9.8-13.5) per 1000 person-years) as compared with Chinese (men: 11.5 (9.3-14.3); women: 6.5 (5.1-8.4) per 1000 person-years). ASIRs for stroke followed a different pattern among the three ethnicities, with Indians having the lowest ASIRs among both DM and non-DM individuals (DM men: 

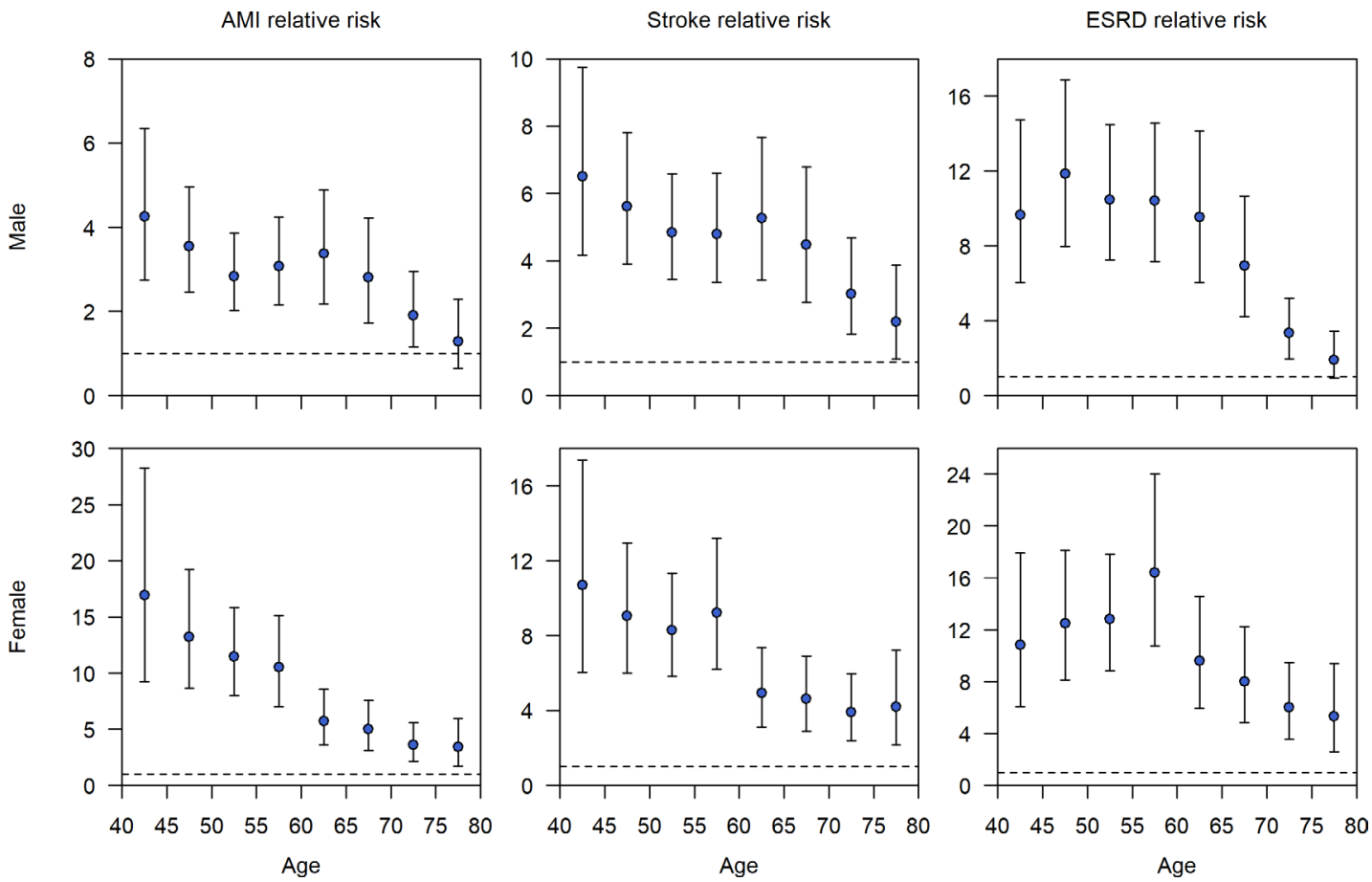

Figure 2 Relative risk of AMI, stroke and ESRD for DM versus non-DM by age and gender for Singaporeans aged 40-79 using estimated DM prevalence and data from Singapore's myocardial infarction, stroke and renal registries. AMI, acute myocardial infarction; DM, diabetes mellitus; ESRD, end-stage renal disease.

7.0 (6.1-8.2); non-DM men: 1.1 (1.0-1.2); DM women: 5.7 (4.8-6.7); non-DM women: $0.6(0.5-0.7)$ per 1000 person-years). For those without DM, Malays had the highest rates (men: 2.4 (2.2-2.7); women: $1.2(1.1-1.3)$ per 1000 person-years), but for those with DM the highest rates were observed in Chinese (men: 11.9 (9.7-14.9); women: 8.4 (6.7-10.7) per 1000 person-years). ASIRs of ESRD were significantly lower than the other two chronic conditions. Malays had the highest ASIRs among those without DM (men: 0.5 (0.4-0.5); women: 0.4 (0.3-0.4) per 1000 person-years), whereas Chinese (men: $0.3(0.3-$ $0.3)$; women: $0.2(0.2-0.2)$ per 1000 person-years $)$ and Indians (men: $0.2(0.2-0.3)$; women: $0.1(0.1-0.2)$ per 1000 person-years) had comparable rates. For those with DM, Malays continue to have the highest ASIRs (men: 3.3 (2.8-4.0); women: $4.2(3.6-5.0)$ per 1000 person-years); however, this time they are preceded by Chinese (men:
2.7 (2.2-3.4); women: 2.3 (1.8-3.0) per 1000 personyears) and subsequently Indians (men: 1.5 (1.3-1.8); women: 1.9 (1.6-2.2) per 1000 person-years).

\section{Projected incidence of sequelae of DM}

When integrating the incidence of AMI, stroke and ESRD-broken down by age, gender, ethnic group and DM status-with projections for the evolving demographic structure of Singapore ${ }^{15}$ and our estimated prevalence of DM, we generated projections of the burden of these sequelae over the next three decades (figure 4). Overlaying the known total and DM incident cases for all sequelae shows a good fit between empirical data and the predictions, with a mean absolute percentage error of $12.6 \%$ for AMI, $20.1 \%$ for stroke and $15.7 \%$ for ESRD. The slight systematic underestimation of projected cases is due to differential incidence rates in those aged 80
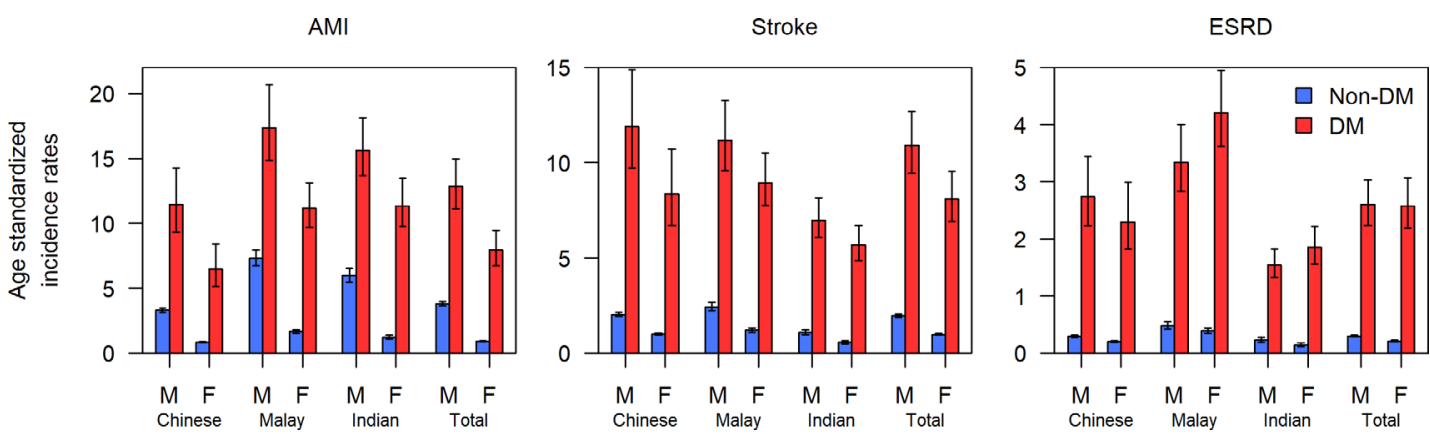

Figure 3 Estimates of age-standardized incidence rates of AMI, stroke and ESRD by ethnicity, gender and DM status for Singaporean adults aged 40-79. AMI, acute myocardial infarction; DM, diabetes mellitus; ESRD, end-stage renal disease; $F$, female; $M$, male. 

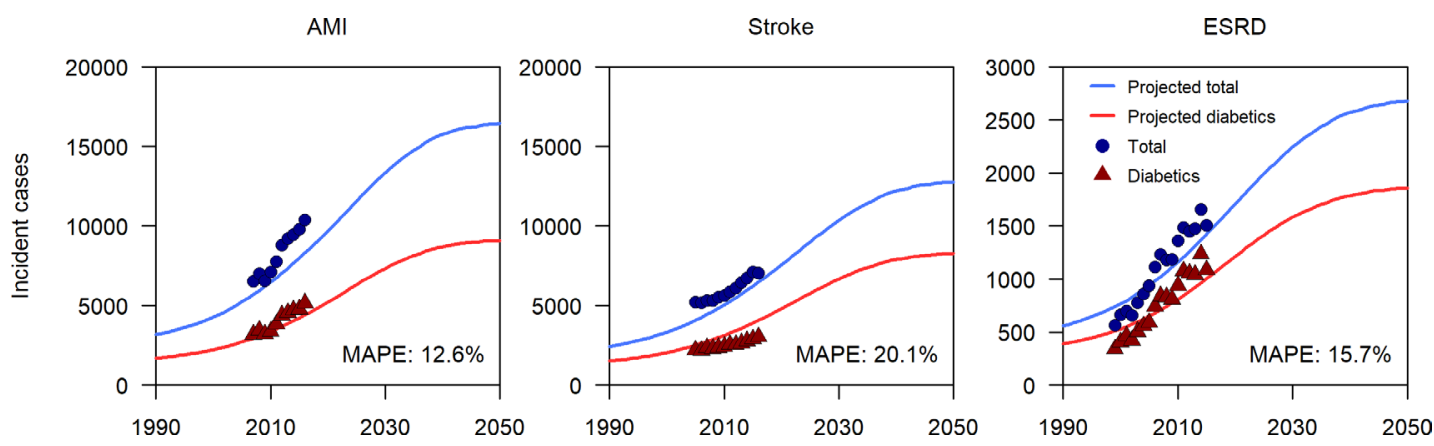

Figure 4 Projected annual number of new cases of AMI, stroke and ESRD in Singapore with MAPE from 1990 to 2050 using population forecasts from a previous modeling study of the number of Singaporeans with DM with constant age-specific prevalence at all time points. The incidence of AMI, stroke and ESRD for age 80 and above is assumed to be equal to that of those aged 75-79. AMI, acute myocardial infarction; DM, diabetes mellitus; ESRD, end-stage renal disease; MAPE, mean absolute percentage error.

and above, which for projection purposes we assumed to have incidence rates of those in the highest age group of 75-79. All three sets of incident cases are expected to increase by a factor of at least $50 \%$ by 2050 (figure 4 ). The annual incidence of AMIs is projected to rise from 9300 (in 2019) to 16400 (in 2050), the number of strokes from 7300 to 12800 , and the number of ESRD cases from 1700 to 2700 . In all of the projections, by 2050 approximately $60 \%$ of the total incident cases will be individuals with DM.

\section{DISCUSSION}

Our findings on age and ethnicity trends are consistent with other international studies reporting differing rates of DM and its sequelae between ethnicities, as well as a decreasing RR as age increases. Tan $e t a l^{26}$ have previously described DM prevalence to be higher in the Malay and Indian ethnicities among Singaporeans. Our results support this observation, with the Chinese of both genders consistently having lower DM prevalence across all age groups by an average of $20 \%$ in comparison with Malays and Indians. A plausible explanation is that Malays and Indians show higher obesity rates in comparison with Chinese ethnicities, ${ }^{27}$ thereby increasing their risk of developing DM. Decreasing RR with age is also consistent with other scientific literature as within the USA; Hillier and Pedula ${ }^{9}$ observed that adults aged 18-44 with DM had an eightfold greater risk of developing any macrovascular complication than non-DM individuals, whereas those aged 45 and above had a fourfold increased risk. Khoury et $a t^{28}$ additionally found a similar trend for stroke, reporting RR values of 5.2 and 2.1 for those below and above age 65, respectively, for an African-American population in 2005 . In our study, the $95 \%$ CI for the RR of AMI and ESRD included 1.0 for men of the oldest age group, implying no significant difference and suggesting that the risk of complications in the elderly was comparable regardless of DM status, and that DM was less of a driver of complications for the elderly. As the highest RRs are observed in younger adults, these results indicate that the early onset of DM causes the greatest increase in the risk of developing sequelae, particularly in ethnic minorities.

By 2050, the annual incidence of sequelae is expected to increase by over $50 \%$, which will increase hospitalization and ambulatory costs significantly, especially among those aged 75 and above. Despite only $10 \%$ of the population having DM, a large proportion of the chronic disease burden will be borne by those with $\mathrm{DM}$ as a comorbidity, as $60 \%$ of incident sequelae cases will have DM. Of the three sequelae, ESRD will have the largest proportion with $\mathrm{DM}$ at $70 \%$ of total incident cases in 2050. ASIRs for all three sequelae show huge differences between those with and without DM, providing the basis behind $10 \%$ of the total population contributing $60 \%$ of the incident cases. Given the rise in cases from 18300 to 31900 per year over a 30 -year horizon, a reasonable strategy to improve population health is to prioritize reducing DM prevalence. From a policy angle, steps must be taken to control the rise in incident sequelae cases to prevent further strain on the healthcare system, or to begin planning to ensure treatments are available population-wide.

Healthcare expenditure in Singapore will rise drastically in the upcoming years as future incidence of these chronic conditions increases. In the local context, $\mathrm{Ng}$ et $a t^{29}$ reported that individuals with DM accrued direct medical costs of US $\$ 1575$ per year, with complications being a strong determinant of cost. Png et $a \vec{l}^{30}$ estimated that by 2050 the economic costs of DM for the working population in Singapore will increase to US $\$ 1.8$ billion, which is the consequence of a rise in DM and its complications. This is supported by a study in Taiwan by Cheng $e t a l,{ }^{31}$ which estimated that the annual healthcare costs of those with DM that developed complications was $\sim$ US $\$ 4200$ as compared with $\sim$ US $\$ 1400$ for those without complications. In light of the excess burden of complications, a multiagency approach is required to safeguard the population from the early onset of DM as well as improve DM management to avoid the development of sequelae associated with DM.

Averting the projected increase will require extensive public health interventions with lifestyle modifications 
on diet and physical activity levels to reduce obesity, a major risk factor. Prevention programs involving counseling of high-risk individuals in USA and Finland ${ }^{32} 33$ reported risk reductions of $58 \%$. In Singapore, a study by Png and Yoong ${ }^{34}$ concluded that both lifestyle modifications and interventions involving metformin are likely to be cost-effective and warrant further investigation. On a larger scale, strategies such as taxation of sugar-sweetened beverages have proven to be efficacious in reducing obesity rates and by proxy the risk of developing DM. ${ }^{35}$ In addition to reducing risks, the revenue from taxation can be directed to local health promotion efforts for dual benefits. ${ }^{36}$ Such sustainable interventions which promote healthy living may be necessary to reduce $\mathrm{DM}$ and sequelae incidence and to alleviate increasing healthcare costs.

Our study had several limitations. First, our fitted models did not account for temporal trends on incidence. The extrapolation of temporal trends to forecast future incident cases for a 30-year time horizon could create errors if the expected trajectory is affected by large policy changes, such as the implementation of an excise tax on sugar-sweetened beverages or changes in migration patterns. Similarly, aggressive management of DM would reduce the risk of developing sequelae and alter the expected trajectory. Due to the small number of cases after stratification by ethnicity and age for the cases below age 40 and above 80 , we chose to omit obtaining risk estimates for them, resulting in a slight underestimation of the future burden. The retrospective study design also means that we were unable to control for possible confounders not collected, such as body mass index, smoking, duration of DM, or family history of DM and the three sequelae. Unavailability of the year of onset or diagnosis of DM resulted in the inability to explore the impact of duration of DM on the risk of developing a sequela. Finally, as the source data contained limited information on predictors and confounders, we were unable to construct and validate a risk engine to predict which individuals in the population would eventually develop sequelae. Despite these limitations, our study still draws useful insights about the differences in chronic disease risk and the potential increasing future healthcare burden and costs.

\section{CONCLUSION}

The total incidence of AMI, stroke and ESRD is expected to increase from 18300 in 2019 to 31900 from DM and its complications in 2050 , where $60 \%$ will be individuals predicted to have DM. As the greatest increments in risk are observed in the younger age groups, it is prudent to step up efforts to prevent early onset of DM in younger adults. A multiagency approach is required to minimize DM prevalence and promote DM management in the upcoming years to combat rising healthcare costs in an aging population such as Singapore. Interventions planned at both the micro-scale and macro-scale which target obesity and other risk factors of DM are recommended to alleviate the healthcare burden in the future as a result of DM and its sequelae.

Acknowledgements We would like to acknowledge the Ministry of Health, Singapore and the National Registry of Diseases Office, Health Promotion Board for the provision of data for this study.

Contributors KWT performed the literature search and data analysis, produced the figures for the manuscript, and was a major contributor in writing the manuscript. BSLD participated in the study design and was a major contributor in writing the manuscript. ARC participated in the study design and was the principal investigator of the study.

Funding The work is supported by the National Research Foundation's Virtual Singapore grant R-608-000-197-281 and the National Medical Research Council's Singapore Population Health Improvement Centre.

Competing interests None declared.

Patient consent for publication Not required.

Provenance and peer review Not commissioned; externally peer reviewed.

Data availability statement Data may be obtained from a third party and are not publicly available. The data that support the findings of this study are available from the National Registry of Diseases Office, Singapore, but restrictions apply to the availability of these data, which were used under license for the current study and so are not publicly available. Data can however be requested from the following website: https://www.nrdo.gov.sg/data-request/staple-dataset-request

Open access This is an open access article distributed in accordance with the Creative Commons Attribution Non Commercial (CC BY-NC 4.0) license, which permits others to distribute, remix, adapt, build upon this work non-commercially, and license their derivative works on different terms, provided the original work is properly cited, appropriate credit is given, any changes made indicated, and the use is non-commercial. See: http://creativecommons.org/licenses/by-nc/4.0/.

\section{ORCID iD}

Ken Wei Tan http://orcid.org/0000-0003-3224-4619

\section{REFERENCES}

1 Beaglehole R, Bonita R, Horton R, et al. Priority actions for the noncommunicable disease crisis. Lancet 2011;377:1438-47.

2 Holman N, Forouhi NG, Goyder E, et al. The Association of Public Health Observatories (APHO) diabetes prevalence model: estimates of total diabetes prevalence for England, 2010-2030. Diabet Med 2011;28:575-82.

3 Waldeyer R, Brinks R, Rathmann W, et al. Projection of the burden of type 2 diabetes mellitus in Germany: a demographic modelling approach to estimate the direct medical excess costs from 2010 to 2040. Diabet Med 2013;30:999-1008.

4 Palacio S, McClure LA, Benavente OR, et al. Lacunar strokes in patients with diabetes mellitus: risk factors, infarct location, and prognosis: the secondary prevention of small subcortical strokes study. Stroke 2014;45:2689-94.

5 Rytter L, Troelsen S, Beck-Nielsen H. Prevalence and mortality of acute myocardial infarction in patients with diabetes. Diabetes Care 1985;8:230-4.

6 van den Brand JAJG. Diabetes mellitus as a cause of end-stage renal disease in Europe: signs of improvement. Clin Kidney $\mathrm{J}$ 2016;9:454-6.

7 Bommer C, Heesemann E, Sagalova V, et al. The global economic burden of diabetes in adults aged 20-79 years: a cost-of-illness study. Lancet Diabetes Endocrinol 2017;5:423-30.

8 Wilmot E, Idris I. Early onset type 2 diabetes: risk factors, clinical impact and management. Ther Adv Chronic Dis 2014;5:234-44.

9 Hillier TA, Pedula KL. Complications in young adults with early-onset type 2 diabetes: losing the relative protection of youth. Diabetes Care 2003;26:2999-3005.

10 Wang L, Gao P, Zhang M, et al. Prevalence and ethnic pattern of diabetes and prediabetes in China in 2013. JAMA 2017;317:2515-23.

11 Tee E-S, Yap RWK. Type 2 diabetes mellitus in Malaysia: current trends and risk factors. Eur J Clin Nutr 2017;71:844-9.

12 Aekplakorn W, Chariyalertsak S, Kessomboon P, et al. Prevalence of diabetes and relationship with socioeconomic status in the Thai 
population: National health examination survey, 2004-2014. J Diabetes Res 2018;2018:1-8.

13 Soewondo P, Ferrario A, Tahapary DL. Challenges in diabetes management in Indonesia: a literature review. Global Health 2013;9:63.

14 Ministry of Health, Singapore. National health survey 2010, 2010.

15 Phan TP, Alkema L, Tai ES, et al. Forecasting the burden of type 2 diabetes in Singapore using a demographic epidemiological model of Singapore. BMJ Open Diabetes Res Care 2014;2:e000012.

16 Low S, Chi LS, Yeoh LY, et al. Long-term diabetes outcomes in multi-ethnic Asians living in Singapore. Diabetes Res Clin Pract 2016;111:83-92.

17 Dreyer G, Hull S, Aitken Z, et al. The effect of ethnicity on the prevalence of diabetes and associated chronic kidney disease. QJM 2009;102:261-9.

18 Ministry of Health, Singapore. Executive summary on national population health survey 2016/17, 2017.

19 International Diabetes Federation. International diabetes Federation Western Pacific region, 2017.

20 The Straits Times. Parliament: Health Minister Gan Kim Yong declares 'war on diabetes'; new task force set up, 2016.

21 Department of Statistics, Singapore. General household survey 2015, 2016.

22 Green PL, Worden K. Bayesian and Markov chain Monte Carlo methods for identifying nonlinear systems in the presence of uncertainty. Philos Trans A Math Phys Eng Sci 2015;373:20140405.

23 National Registry of Diseases Office. Available: https://www.nrdo. gov.sg/

24 Plummer M. JAGS: a program for analysis of Bayesian graphical models using Gibbs sampling. Proceedings of the third international workshop on distributed statistical computing, 2003.

25 RC Team. R: a language and environment for statistical computing, 2013.
26 Tan KHX, Tan LWL, Sim X, et al. Cohort profile: the Singapore multiethnic cohort (MEC) study. Int J Epidemiol 2018;47:699.

27 Lee WR. The changing demography of diabetes mellitus in Singapore. Diabetes Res Clin Pract 2000;50:S35-9.

28 Khoury JC, Kleindorfer D, Alwell K, et al. Diabetes mellitus: a risk factor for ischemic stroke in a large biracial population. Stroke 2013:44:1500-4.

$29 \mathrm{Ng}$ CS, Toh MPHS, Ko Y, et al. Direct medical cost of type 2 diabetes in Singapore. PLoS One 2015;10:e0122795.

30 Png ME, Yoong J, Phan TP, et al. Current and future economic burden of diabetes among working-age adults in Asia: conservative estimates for Singapore from 2010-2050. BMC Public Health 2016;16.

31 Cheng S-W, Wang C-Y, Chen J-H, et al. Healthcare costs and utilization of diabetes-related complications in Taiwan. Medicine 2018;97:e11602.

32 Tuomilehto J, Lindström J, Eriksson JG, et al. Prevention of type 2 diabetes mellitus by changes in lifestyle among subjects with impaired glucose tolerance. N Engl J Med 2001;344:1343-50.

33 Knowler WC, Barrett-Connor E, Fowler SE, et al. Reduction in the incidence of type 2 diabetes with lifestyle intervention or metformin. N Engl J Med 2002;346:393-403.

34 Png ME, Yoong JS-Y. Evaluating the cost-effectiveness of lifestyle modification versus metformin therapy for the prevention of diabetes in Singapore. PLoS One 2014;9:e107225.

35 Nakhimovsky SS, Feigl AB, Avila C, et al. Taxes on sugarsweetened beverages to reduce overweight and obesity in middle-income countries: a systematic review. PLoS One 2016;11:e0163358.

36 Brownell KD, Farley T, Willett WC, et al. The public health and economic benefits of taxing sugar-sweetened beverages. $N$ Engl $J$ Med 2009;361:1599-605. 